\title{
Erratum to: Pulse Waves
}

\author{
Paolo Salvi
}

(C) Springer International Publishing 2017

P. Salvi, Pulse Waves, DOI 10.1007/978-3-319-40501-8

\section{DOI 10.1007/978-3-319-40501-8_9}

An error in the production process unfortunately led to publication of this book prematurely, before incorporation of the final corrections. The version supplied here has been corrected and approved by the author.

The updated online version of the original book can be found at http://dx.doi.org/10.1007/978-3-319-40501-8 